\title{
Pulmonary Dual Hemodynamic Changes in Severe COPD Patients: A Quantitative Study Using Low-Dose CT Lung Perfusion Scan
}

\author{
Jin Fang ${ }^{1}$, Honglin $\mathrm{Li}^{2}$, Minjie Liang ${ }^{1}$, Dabiao Deng ${ }^{3}$ and Quan Zhou ${ }^{2, *}$ \\ ${ }^{1}$ Medical Imaging Center, The First Affiliated Hospital, Jinan University, Guangzhou, China \\ ${ }^{2}$ Department of Radiology, The Third Affiliated Hospital of Southern Medical University, Guangzhou, China \\ ${ }^{3}$ Department of Imaging Center, Guangdong 999 Brain Hospital, Guangzhou, China \\ "Corresponding author: Quan Zhou, Department of Radiology, The Third Affiliated Hospital of Southern Medical University, 510630, Guangzhou, China. Tel: +86-18926121318, \\ Fax: +86-2038688900, Email: oimaging@jnu.edu.cn
}

Received 2018 March 26; Revised 2018 September 15; Accepted 2018 September 24.

\begin{abstract}
Background: Computed tomography (CT) lung perfusion scan could be used to evaluate regional, morphologic, and functional changes in chronic obstructive pulmonary disease (COPD) noninvasively. However, the dual hemodynamic changes in severe COPD patients have not been studied quantitatively using CT lung perfusion scan.

Objectives: To determine the dual hemodynamic changes quantitatively in patients with severe COPD by using low-dose CT lung perfusion scan.

Patients and Methods: Fifteen patients with severe COPD (global initiative for chronic obstructive lung disease [GOLD] class IV) and 31 controls were enrolled. All participants received low-dose CT lung perfusion scan using a Toshiba 320-detector row dynamic volumetric CT. The perfusion parameters including pulmonary artery flow (PAF), bronchial artery flow (BAF), perfusion index [PI $=\mathrm{PAF} /(\mathrm{PAF}+\mathrm{BAF})]$ and time to peak (TTP) of the time density curve were generated and compared between the impaired lung parenchyma in the COPD group and normal lung parenchyma in the control group.

Results: The PAF and PI values in the COPD group were significantly lower than that in the control group $(\mathrm{P}<0.001, \mathrm{P}<0.001)$, while the BAF value was significantly higher $(\mathrm{P}<0.001)$. The TTP of impaired lung parenchyma was significantly prolonged compared to the normal lung parenchyma $(\mathrm{P}<0.001)$.

Conclusion: Patients with severe COPD have distinct changes in pulmonary dual hemodynamics. Low-dose lung perfusion scan using a 320-detector row dynamic volumetric CT could be used to evaluate the pulmonary dual hemodynamics.
\end{abstract}

Keywords: Chronic Obstructive Pulmonary Disease, Dynamic Volumetric CT, Perfusion

\section{Background}

Chronic obstructive pulmonary disease (COPD) is a multicomponent disease containing a combination of small airway disease (bronchiolitis) and parenchyma destruction (emphysema), as characterized by incompletely reversible airflow limitation (1). COPD has been currently the fourth leading cause of death worldwide (2). As the global population ages, the social and family burden of COPD are increasing continuously (3).

Pulmonary function test (PFT), especially spirometry, is the most widely used clinical test for COPD, but it can not determine morphological abnormalities, regional dysfunction and pathological changes (4). Moreover, allowing for the risk of bullae ruptures, COPD patients with the presence of pulmonary bulla often can not perform PFT. However, computed tomography (CT) lung perfusion scan could be used to evaluate regional, morphologic, and functional changes in COPD noninvasively $(5,6)$. In previous studies, the scan range of 16-slice or 64-slice multidetector row CT is incapable to cover the whole lung in a single rotation, thus multiple scanning would lead to the increase of radiation dose (7-9). Furthermore, the bronchial artery hemodynamic abnormalities that are common in COPD patients and correlate closely with life-threatening bleeding from the airways have not been studied quantitatively using CT lung perfusion scan (5, 6, 8, 9). A 320-detector row CT with the dual input model has the capacity to evaluate the dual hemodynamics of organs. Meanwhile, it reduces radiation dose by covering the whole lung in a single rotation $(10,11)$. To the best of our knowledge, there have been no studies investigating dual hemodynamic changes in severe COPD patients using CT lung perfusion scan. 


\section{Objectives}

In this prospective study, we performed low-dose CT lung perfusion scan using a 320-detector row dynamic volumetric CT in severe COPD patients and healthy subjects. The objective of this study was to determine dual hemodynamic changes quantitatively in patients with severe COPD by using low-dose CT lung perfusion scan.

\section{Patients and Methods}

\subsection{Study Population}

This study was approved by the Medical Ethics Committee of Jinan University, and written informed consent was obtained from each participant. Thirty-five subjects with suspicious lung nodules during physical examination and 20 patients with severe COPD were enrolled between January 1, 2015 and January 31, 2016. The inclusion criteria of severe COPD were: post-bronchodilator forced expiratory volume in one second $\left(\mathrm{FEV}_{1}\right) /$ forced vital capacity (FVC) < $70 \%, \mathrm{FEV}_{1}<30 \%$ predicted (12). Major exclusion criteria were: (1) coexistence with other pulmonary diseases such as pulmonary tuberculosis, pleural effusion and bronchial asthma; (2) coexistence with heart failure or respiratory failure (3) contraindication for enhanced CT scan, such as allergy to the iodine agent, and renal failure. Finally, fifteen severe COPD patients (nine males and six females; mean age, $54.5 \pm 15.5$ years) and 31 healthy subjects ( 16 males and 15 females; mean age, $64.9 \pm 10.7$ years) were included in this study.

\subsection{Imaging Techniques}

CT lung perfusion scans were performed in all participants by using a 320-detector row CT scanner (Aquilion One; Toshiba Medical Systems, Tochigi, Japan). In order to minimize the effect of cardiac drugs on vasodilatation, all patients included were required not to take medicine on the day before the CT examination. Baseline chest CT scanning was performed. The imaging parameters of perfusion scan are summarized in Table 1 . Restraining bands were placed around the subcostal abdominal wall to limit respiratory motions, and all participants were required to breathe gently during the scan. The density of regions of interest (ROIs) was measured two seconds after the injection of contrast medium, containing 15 volumes with a 2$\mathrm{s}$ interval. Image data of each volume was reconstructed with $0.5-\mathrm{mm}$ slice thickness and $0.5-\mathrm{mm}$ spacing. The scan coverage was $160 \mathrm{~mm}$, thus generating 160/0.5 = $320 \mathrm{im}$ ages per volume. There were 15 volumes in each perfusion

\begin{tabular}{|c|c|}
\hline Variables & Value \\
\hline \multicolumn{2}{|c|}{ Scan parameters } \\
\hline Number of detector rows & 320 \\
\hline Craniocaudal coverage & $160 \mathrm{~mm}$ \\
\hline Collimation & $0.5 \mathrm{~mm}$ \\
\hline Tube voltage & $80 \mathrm{kV}$ \\
\hline Tube current & $70 \mathrm{~mA}$ \\
\hline Gantry rotation time & $0.5 \mathrm{~s}$ \\
\hline Matrix & $512 \times 512$ \\
\hline Field of view & $300 \mathrm{~mm} \times 350 \mathrm{~mm}$ \\
\hline Thickness & $0.5 \mathrm{~mm}$ \\
\hline Scan center & Hilum of lung \\
\hline \multicolumn{2}{|c|}{ Contrast agent } \\
\hline Iodine concentration & $370 \mathrm{mg} / \mathrm{mL}$ \\
\hline Injection rate & $7 \mathrm{mLs}^{-1}$ \\
\hline Total dose & $56 \mathrm{~mL}$ \\
\hline Saline flush & $50 \mathrm{~mL}$ with $7 \mathrm{ml} \mathrm{s}^{-1}$ \\
\hline Scan & Every $2 \mathrm{~s}$ for $30 \mathrm{~s}$ \\
\hline
\end{tabular}

scan, resulting in a total of $15 \times 320=4800$ images. Effective radiation dose was calculated by using the following equation: Effective radiation dose $(\mathrm{mSv})=$ dose-length product $(\mathrm{DLP})(\mathrm{mGy} . \mathrm{cm}) \times 0.014\left(\mathrm{mSv} \mathrm{mGy}^{-1} \mathrm{~cm}^{-1}\right)$. The DLP and conversion coefficient was proposed by the International Commission on Radiological Protection (13). The plain scan and perfusion DLP were $26.6 \mathrm{mGy.cm}$ and 260.4 mGy.cm respectively, the total effective radiation dose for each patient was $4.02 \mathrm{mSv}$.

\subsection{Image Analysis}

All CT perfusion images were transferred to the CT processing workstation. First, the images were adjusted automatically with body registration software (Body Registration; Toshiba Medical System, Japan). The body perfusion software V4.74 (Toshiba Medical Systems) was applied for perfusion data analysis, which applied the dual-input maximum slope analysis method. The window width range was set as -1000-0 HU to eliminate the interference of bone and large vessels. For each subject, four regions of interest (ROI) were drawn on the main trunk of the pulmonary artery, the descending aorta, the left atrium and the lung parenchyma to generate four time-density curves (TDCs) (Figure 1). The time to peak (TTP) of each TDC was recorded. The ROIs were manually placed in the pulmonary artery trunk and the aorta at the level of the pulmonary hilum to 
generate the TDCs representing the PA input function and the BA input function respectively, and the left atrium was selected to distinguish body circulation and pulmonary circulation as described by Yuan et al. (14). Then, the functional maps were generated, including pulmonary artery flow (PAF), bronchial artery flow (BAF) and perfusion in$\operatorname{dex}[\mathrm{PI}=\mathrm{PAF} /(\mathrm{PAF}+\mathrm{BAF})]$. The parameters are expressed in milliliter per $100 \mathrm{~mL}$ per min.

On parametric images, ROIs were manually drawn on the bilateral peripheral zone of the lung on three slices including the aortic arch slice, the carina slice, and the slice $2-3 \mathrm{~cm}$ above the top of the diaphragm. ROIs were chosen to keep away from the surrounding vessels and soft tissues. For each participant, each pulmonary perfusion index was the average of all ROIs on the three slices. Each participant was assessed independently by M.L and D.D, each with 5 and 8 years of experience in chest CT diagnosis.

\subsection{Statistical Analysis}

Data are expressed as mean \pm standard deviation (SD). Interobserver reproducibility of the perfusion parameters was evaluated by using the intraclass correlation coefficient (ICC). The Kolmogorov-Smirnov test and Levene's test were used to evaluate the normal distribution and homogeneity of variance of all perfusion indexes. The independent sample $t$-test was applied to compare the lung perfusion indexes and the TTP of TDCs between the COPD group and the control group. Statistical analysis was performed by using SPSS 19.0 software (SPSS Inc., Chicago, IL, USA). P < 0.05 was considered to indicate a significant difference.

\section{Results}

\subsection{Pulmonary Perfusion Parameters}

The ICC for PAF, BAF, and PI were 0.952 (95\% confidence interval [CI]: 0.922 - 0.968), 0.943 (95\% CI: 0.915 - 0.963), and 0.939 (95\% CI: 0.907-0.956) respectively, suggesting a good interobserver measurement reproducibility. Two radiologists' measurements of $\mathrm{PAF}, \mathrm{BAF}$, and $\mathrm{PI}$ in each patient was averaged to generate the final value of perfusion indexes. The pulmonary perfusion indexes in the control group and COPD group are shown in Table 2. The PAF and PI values in the COPD group were significantly lower than that in the control group $(\mathrm{P}<0.001, \mathrm{P}<0.001)$, while the $\mathrm{BAF}$ value in the COPD group was significantly higher than that in the control group $(\mathrm{P}<0.001)$. PAF, BAF, and PI parametric maps of a healthy subject and a COPD patient are shown in Figure 2.

\begin{tabular}{lccc}
\hline \multicolumn{1}{l}{ Table 2. Perfusion Parameters of the Control Group and COPD Group ${ }^{\mathrm{a}}$} & \\
\hline & $\begin{array}{c}\text { PAF } \\
(\mathbf{m L} / \mathbf{m i n} / \mathbf{1 0 0 m L})\end{array}$ & $\begin{array}{c}\text { BAF } \\
(\mathbf{m L} . \mathbf{m i n} / \mathbf{1 0 0 m L})\end{array}$ & PI (\%) \\
\hline Control group & $102.43 \pm 71.68$ & $2.28 \pm 0.29$ & $98.76 \pm 1.86$ \\
\hline COPD group & $76.69 \pm 24.90$ & $20.70 \pm 23.72$ & $82.22 \pm 6.63$ \\
P value & $<0.001$ & $<0.001$ & $<0.001$ \\
\hline $\begin{array}{l}\text { Abbreviations: } \\
\text { monary disease; PAF, pulmonary artery flow; PI, perfusion index; SD, standard } \\
\text { deviation. }\end{array}$ & Bafonchial artery flow; COPD, chronic obstructive pul- \\
a Values are expressed as mean \pm SD.
\end{tabular}

\subsection{Time to Peak}

The TTP of the pulmonary parenchyma TDC, the pulmonary artery TDC, the aorta TDC and the left atrium TDC are shown in Table 3. The TTP of lung parenchyma in COPD patients was significantly prolonged than that in healthy subjects ( $\mathrm{P}<0.001)$. The TDCs of a healthy subject and a COPD patient are shown in Figure 3. No significant difference was found in TTP of the aorta TDC, the pulmonary artery TDC and the left atrium TDC between the two groups $(\mathrm{P}>0.05)$.

\section{Discussion}

In the present study, we demonstrated significant increase of BAF and decrease of PAF and PI in severe COPD patients, and confirmed the feasibility of low-dose CT lung perfusion scan to evaluate the pulmonary dual hemodynamics.

PFT, particularly spirometry, is the cornerstone for diagnosis and classification in patients with COPD (15). The diagnosis of severe COPD is made when $\mathrm{FEV}_{1} / \mathrm{FVC}<70 \%$, and $\mathrm{FEV}_{1}<30 \%$ are predicted (12). The patients with respiratory failure or heart failure sometimes are too weak to perform PFT. Meanwhile, pulmonary bullae are not uncommon in severe COPD patients. Pulmonary function tests take the risk of bullae rupture for these patients. Thus, a non-invasive imaging modality is highly desirable for accurate evaluation of pulmonary dysfunction in severe COPD. In Guan's study, pulmonary perfusion parameters were positively correlated with $\mathrm{FEV}_{1}, \mathrm{FEV}_{1} \%$ predicted, FVC and $\mathrm{FEV}_{1} / \mathrm{FVC}$ (5). Under this circumstance, CT lung perfusion scan is a preferable diagnostic tool in detecting pulmonary dysfunction.

However, the greatest limitation for CT perfusion scanning, particularly serial CT scanning, is the risk of ionizing radiation (7). To overcome this limitation, low-dose CT scanning is increasingly utilized (16). In studies performed by Alkadhi et al. (17) and Hosch et al. (18), CT perfusion scan 

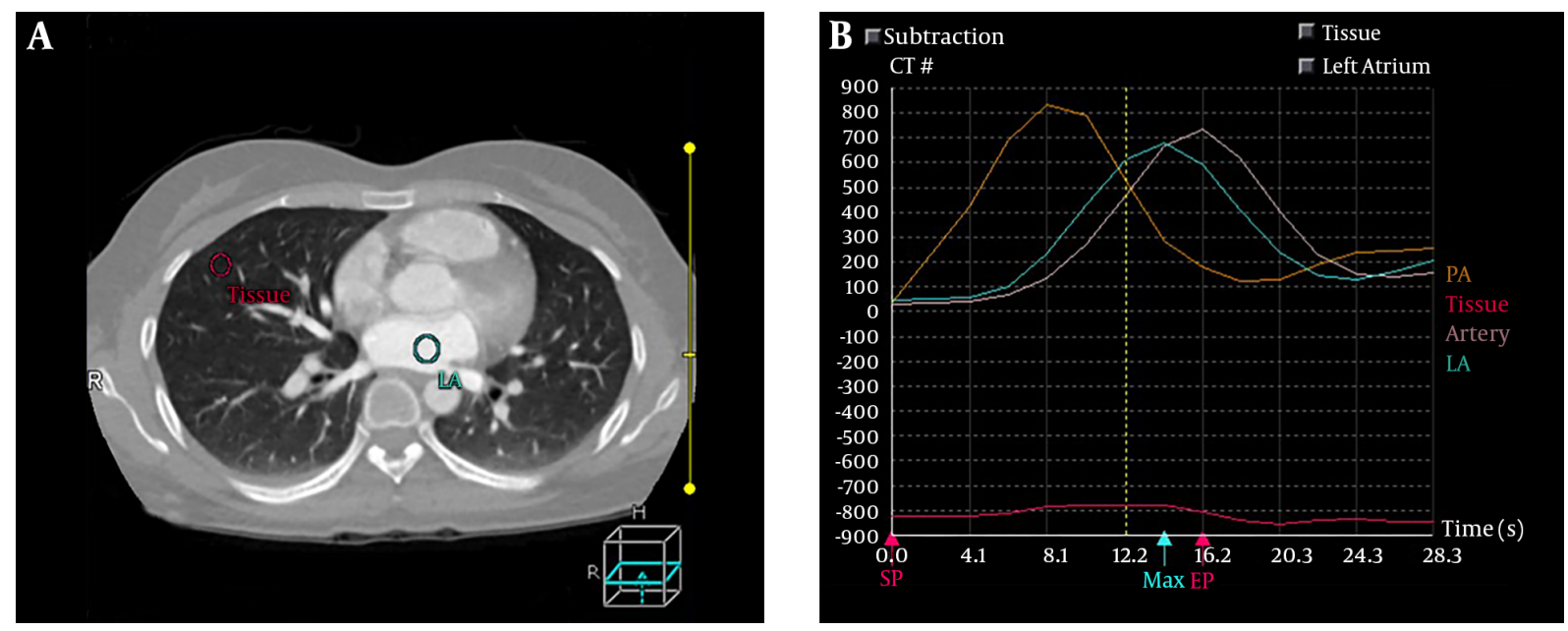

Figure 1. CT perfusion image in a 56-year-old healthy female. A, Regions of interest(ROIs). B, Four time-density curves (TDCs), among which the yellow one represents TDC of the pulmonary artery (PA), the blue one represents TDC of the left atrium (LA), pink one represents TDC of the descending aorta (artery), and the red one represents TDC of pulmonary tissue (tissue).
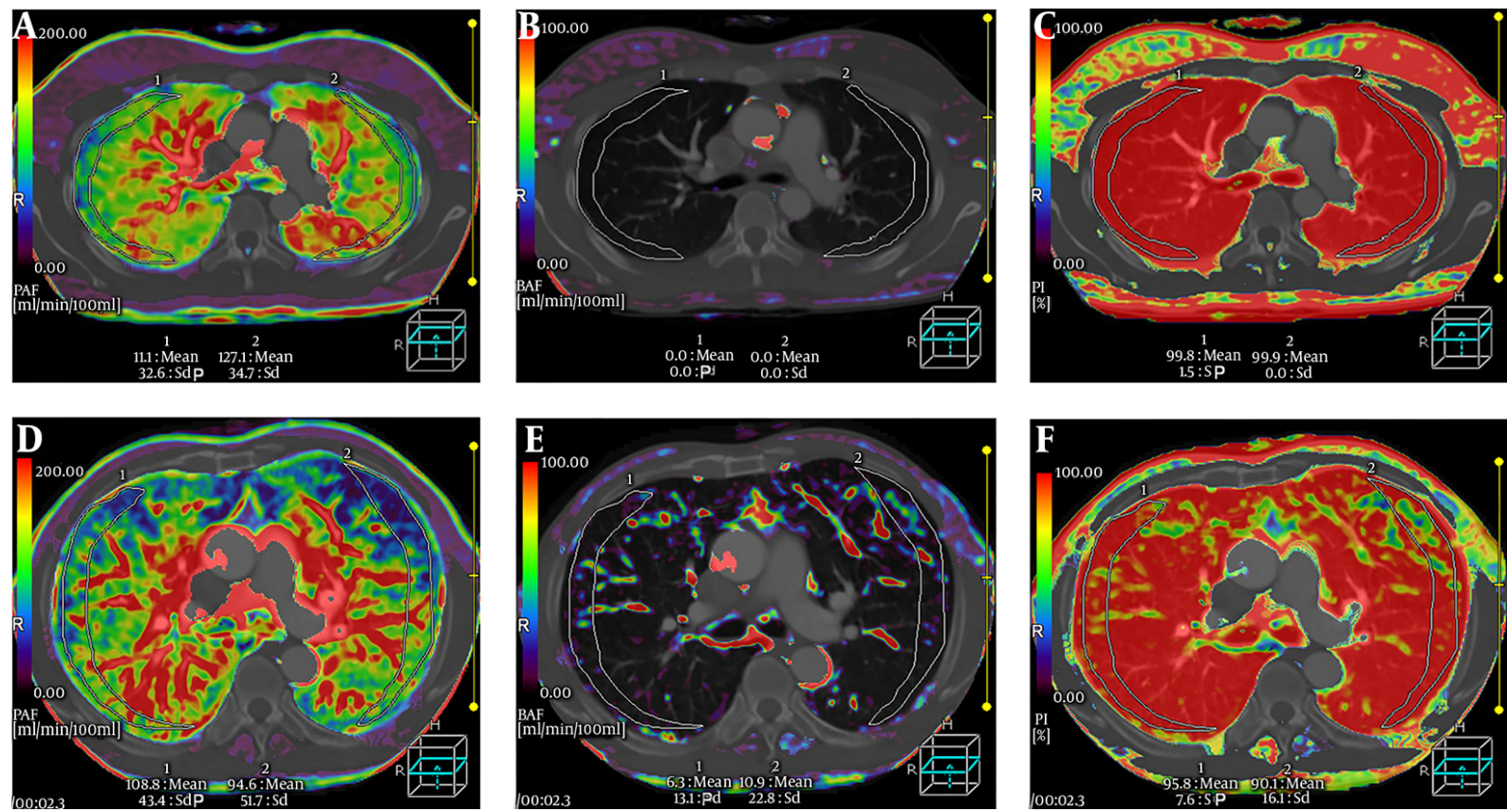

Figure 2. A-C, Pulmonary artery flow (PAF), bronchial artery flow (BAF), perfusion index (PI) parametric maps of a 70-year-old healthy man. A, PAF is in homogeneous distribution. B, BAF is very little. C, PI is also homogeneous. D-F, PAF, BAF, and PI parametric maps of a 69-year-old male patient with chronic obstructive pulmonary disease (COPD). D, Heterogeneously decreased PAF. E, Heterogeneously increased BAF. F, Heterogeneously reduced PI.

using the adaptive iterative dose reduction (AIDR) reconstruction algorithm and the $100 \mathrm{KV}$ tube voltage reduced the scan dosage to about $80 \%$ of conventional chest CT scan, and the image quality was good enough for the diagnosis. In our study, the 320-detector row CT using the 160 $\mathrm{mm}$ z-coverage could obtain the volumetric information in a single rotation. All dose saving technologies including AIDR reconstruction algorithm, the shortest tube rotation time, and the post-processing techniques are ready for use. Meanwhile, the slight lower voltage $(80 \mathrm{KV})$ was uti- 


\begin{tabular}{lccccc}
\hline Table 3. TTP of the Control Group and COPD Group & & & \\
\hline & PA & LA & BA & Lung parenchyma \\
\hline Control group & $8.34 \pm 1.00$ & $13.59 \pm 1.51$ & $16.27 \pm 2.37$ & $11.33 \pm 1.69$ \\
COPD group & $8.46 \pm 1.41$ & $14.03 \pm 2.46$ & $17.07 \pm 3.24$ & 0.47 & $14.86 \pm 1.28$ \\
Pvalue & 0.80 & 0.60 & $<.001$ \\
\hline
\end{tabular}

Abbreviations: BA, bronchi artery; COPD, chronic obstructive pulmonary disease; LA, left atrium; PA, pulmonary artery; SD, standard deviation; TTP, time to peak. ${ }^{\text {a }}$ Values are expressed as mean $\pm \mathrm{SD}$.
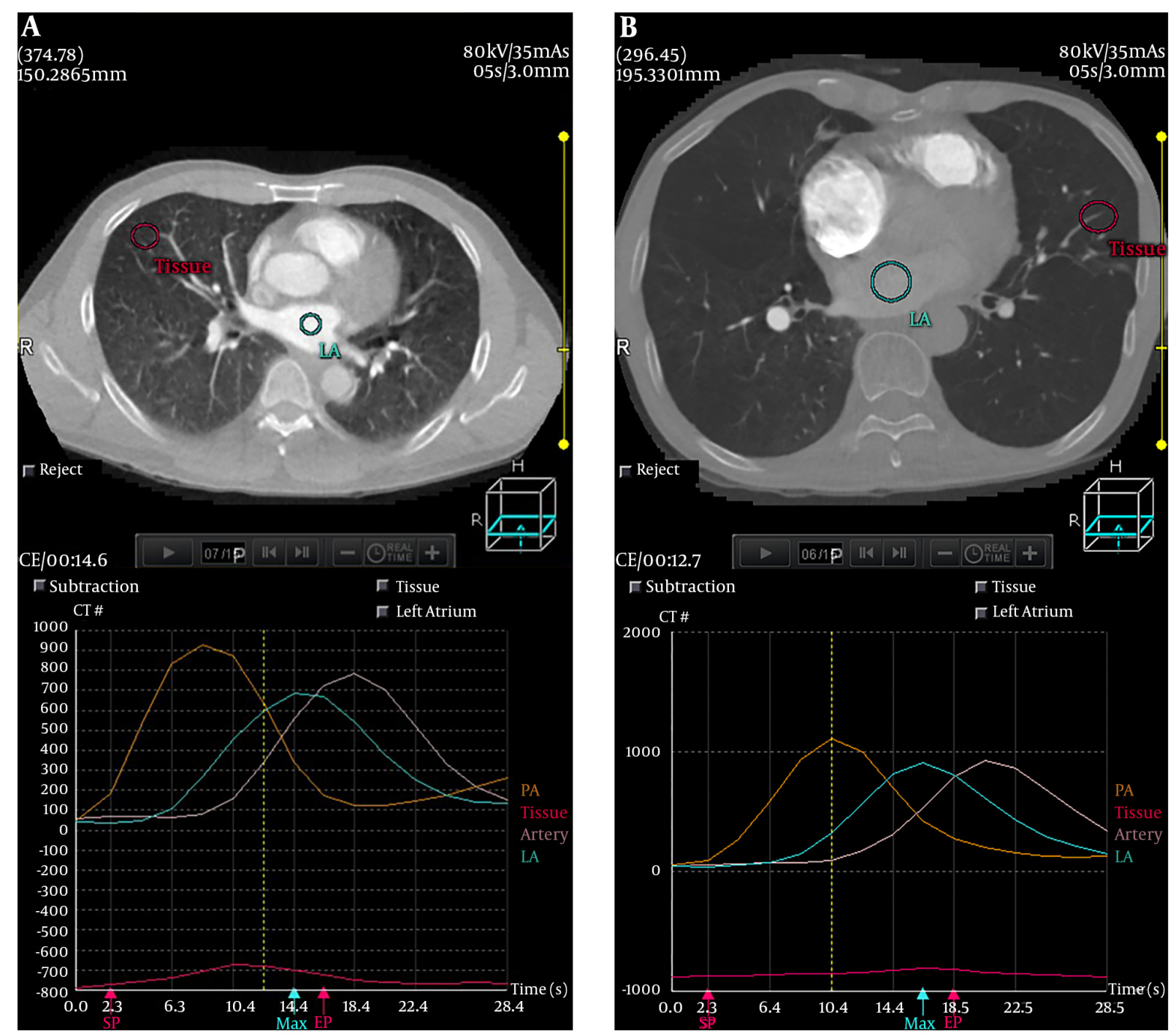

Figure 3. A, Time-density curves (TDC) of a 54-year-old healthy male. The time to peak (TTP) of pulmonary parenchyma TDC (red) is $12.4 \mathrm{~s}$. B, TDCs of a chronic obstructive pulmonary disease (COPD) patient. The TTP of pulmonary parenchyma TDC (red) is $16.4 \mathrm{~s}$.

lized in our study. Thus, the radiation dose in our study was lower than that of Alkadhi et al. (17) and Hosch et al. (18) studies. Meanwhile, the image quality was sufficient to demonstrate the distinct changes of pulmonary dual hemodynamics in severe COPD patients.

Previously, CT perfusion scan was mainly used to assess pulmonary artery hemodynamics in COPD patients ( 8 , 19). Nevertheless, lung is a dual blood supply organ, and 
bronchial artery hemodynamic abnormalities are common in COPD (20). To make it clear, we used the dual input model to evaluate the pulmonary and systemic circulation quantitatively. In the present study, the patients with severe COPD had a lower PAF than normal subjects. This is consistent with findings of Fan et al. (19), Ogasawara et al. (21) and Ley-Zaporozhan and van Beek (22). They utilized the MR, CT or SPECT to qualitatively assess lung perfusion, and all showed that PAF was decreased in COPD patients. This phenomenon can be explained by several factors. In COPD patients, ventilation is damaged due to small airway obstruction and pulmonary parenchyma destruction. The hypoxic vasoconstriction in lung parenchyma with reduced ventilation leads to decrease of PAF (23). Besides, pulmonary hypertension caused by alveolar expansion and capillary bed destruction also result in decrease of PAF (24).

In this study, the patients with severe COPD had a higher BAF. The bronchial microvasculature broadly interconnects with the pulmonary arterial circulation via bronchial and alveolar capillary beds (24). When the pulmonary blood flow decreases, the bronchial blood circulation respond with compensatory increase (24). This would result in an increase of BAF. PI is related to the proportion of the pulmonary and systematic circulation, which directly reflects the change of pulmonary dual hemodynamics. In the present study, the decreased PI indicated the increased proportion of systematic circulation. Bronchial artery dilatation arising from the increased systematic circulation would add the risk of life-threatening bleeding from the airways (25). Therefore, both BAF and PI could be used as biomarkers of risk event in COPD patients.

Time to peak (TTP) is defined as the time to reach the maximum value of contrast material concentration (26). Recently, TTP has been proved to be a surrogate parameter to increase the quantitative accuracy of CT perfusion imaging in animal models of bronchial occlusion (27). The TTP of lung parenchyma in COPD patients was significantly prolonged compared to healthy subjects, which was in accordance with the finding of Remy-Jardin et al. (28). The TTP prolongation may be explained by a significant increase in pulmonary blood flow resistance resulting from the thickening microvascular wall and destructive capillary beds.

This study still had several limitations. First, specific PFT data of each COPD patient was unavailable for us in this study. The correlation between PFT parameters and pulmonary dual hemodynamic indexes (PAF, BAF and PI) should be validated in follow up study. Second, the sam- ple size of this research was relatively small. Further studies with a large cohort of patients with COPD in different stages is needed to confirm the correlation between CT pulmonary perfusion parameters and the severity of COPD. Third, our post-processing software only permitted the manual selection of ROI in a single image plane. A 3D ROI technique which can cover all destructive lung parenchyma should be developed to improve the accuracy of perfusion indexes evaluation.

In conclusion, low-dose CT lung perfusion scanning using a 320-row $\mathrm{CT}$ could be used to quantitatively evaluate the pulmonary dual hemodynamics in severe COPD patients.

The authors declare no conflict of interest.

\section{Footnotes}

Authors' Contributions: Jin Fang conceived and perfomed all of the experiments, analyzed the data and wrote the paper. Honglin Li contributed to experimental design and paper discussion. Minjie Liang and Dabiao Deng contributed to the data analyses. Quan Zhou conceived and directed the research.

Financial Disclosure: None declared.

Funding/Support: This research was supported by the National Natural Science Foundation of China (grant numbers: 81471659 and 81630046 ).

\section{References}

1. Calverley PM, Walker P. Chronic obstructive pulmonary disease. Lancet. 2003;362(9389):1053-61. [PubMed: 14522537].

2. Chen H, Chen RC, Guan YB, Li W, Liu Q, Zeng QS. Correlation of pulmonary function indexes determined by low-dose MDCT with spirometric pulmonary function tests in patients with chronic obstructive pulmonary disease. AJR Am J Roentgenol. 2014;202(4):711-8. doi: 10.2214/AJR.12.10501. [PubMed: 24660696].

3. Mannino DM, Buist AS. Global burden of COPD: Risk factors, prevalence, and future trends. Lancet. 2007;370(9589):765-73. doi: 10.1016/S0140-6736(07)61380-4. [PubMed: 17765526].

4. Heussel CP, Herth FJ, Kappes J, Hantusch R, Hartlieb S, Weinheimer $\mathrm{O}$, et al. Fully automatic quantitative assessment of emphysema in computed tomography: Comparison with pulmonary function testing and normal values. Eur Radiol. 2009;19(10):2391-402. doi: 10.1007/s00330-009-1437-z. [PubMed: 19458953].

5. Guan Y, Xia Y, Fan L, Liu SY, Yu H, Li B, et al. Quantitative assessment of pulmonary perfusion using dynamic contrast-enhanced $\mathrm{CT}$ in patients with chronic obstructive pulmonary disease: Correlations with pulmonary function test and CT volumetric parameters. Acta Radiol. 2015;56(5):573-80. doi: 10.1177/0284185114535208. [PubMed: 24917608].

6. Hoffman EA, Lynch DA, Barr RG, van Beek EJ, Parraga G, Iwpfi Investigators . Pulmonary CT and MRI phenotypes that help explain chronic pulmonary obstruction disease pathophysiology and outcomes. 
J Magn Reson Imaging. 2016;43(3):544-57. doi: 10.1002/jmri.25010. [PubMed: 26199216]. [PubMed Central: PMC5207206].

7. Sodickson A, Baeyens PF, Andriole KP, Prevedello LM, Nawfel RD, Hanson R, et al. Recurrent CT, cumulative radiation exposure, and associated radiation-induced cancer risks from CT of adults. Radiology. 2009;251(1):175-84. doi:10.1148/radiol.2511081296. [PubMed: 19332852].

8. Fan L, Xia Y, Guan Y, Zhang TF, Liu SY. Characteristic features of pulmonary function test, CT volume analysis and MR perfusion imaging in COPD patients with different HRCT phenotypes. Clin Respir J. 2014;8(1):45-54. doi: 10.1111/crj.12033. [PubMed: 23711228].

9. Page M, Nandurkar D, Crossett MP, Stuckey SL, Lau KP, Kenning N, et al. Comparison of $4 \mathrm{~cm}$ Z-axis and $16 \mathrm{~cm}$ Z-axis multidetector CT perfusion. Eur Radiol.2010;20(6):1508-14. doi:10.1007/s00330-009-1688-8. [PubMed: 20013273].

10. Kanda T, Yoshikawa T, Ohno Y, Fujisawa Y, Kanata N, Yamaguchi M, et al. Perfusion measurement of the whole upper abdomen of patients with and without liver diseases: Initial experience with 320-detector row CT. Eur JRadiol. 2012;81(10):2470-5. doi:10.1016/j.ejrad.2011.10.009. [PubMed: 22055684].

11. Motosugi U, Ichikawa T, Sou H, Morisaka H, Sano K, Araki T. Multiorgan perfusion $\mathrm{CT}$ in the abdomen using a 320-detector row CT scanner: Preliminary results of perfusion changes in the liver, spleen, and pancreas of cirrhotic patients. Eur J Radiol. 2012;81(10):2533-7. doi: 10.1016/j.ejrad.2011.11.054. [PubMed: 22227262].

12. Global Initiative for Chronic Obstructive Lung Disease (GOLD). Global strategy for the diagnosis, management, and prevention of chronic obstructive pulmonary disease. 2008.

13. Valentin J; International Commission on Radiation Protection Managing patient dose in multi-detector computed tomography(MDCT). ICRP publication 102. Ann ICRP. 2007;37(1):1-79. iii. [PubMed: 18069128].

14. Yuan X, Zhang J, Ao G, Quan C, Tian Y, Li H. Lung cancer perfusion: Can we measure pulmonary and bronchial circulation simultaneously? Eur Radiol. 2012;22(8):1665-71. doi: 10.1007/s00330-012-2414-5. [PubMed: 22415414].

15. Soriano JB, Maier WC, Visick G, Pride NB. Validation of general practitioner-diagnosed COPD in the UK general practice research database. Eur J Epidemiol. 2001;17(12):1075-80. [PubMed: 12530765].

16. Tschirren J, Hoffman EA, McLennan G, Sonka M. Intrathoracic airway trees: Segmentation and airway morphology analysis from low-dose CT scans. IEEE Trans Med Imaging. 2005;24(12):1529-39. doi: 10.1109/TMI.2005.857654. [PubMed: 16353370]. [PubMed Central: PMC1851666].

17. Alkadhi H, Stolzmann P, Scheffel H, Desbiolles L, Baumuller S, Plass A, et al. Radiation dose of cardiac dual-source CT: The effect of tailoring the protocol to patient-specific parameters. Eur J Radiol. 2008;68(3):385-91. doi: 10.1016/j.ejrad.2008.08.015. [PubMed: 18976876].

18. Hosch W, Stiller W, Mueller D, Gitsioudis G, Welzel J, Dadrich M, et al. Reduction of radiation exposure and improvement of image quality with BMI-adapted prospective cardiac computed tomography and iterative reconstruction. Eur J Radiol. 2012;81(11):3568-76. doi: 10.1016/j.ejrad.2011.06.055. [PubMed: 21784592].

19. Fan L, Xia Y, Guan Y, Yu H, Zhang TF, Liu SY, et al. Capability of differentiating smokers with normal pulmonary function from COPD patients: A comparison of CT pulmonary volume analysis and MR perfusion imaging. Eur Radiol. 2013;23(5):1234-41. doi: 10.1007/s00330-0122729-2. [PubMed: 23263604].

20. Zanini A, Chetta A, Imperatori AS, Spanevello A, Olivieri D. The role of the bronchial microvasculature in the airway remodelling in asthma and COPD. Respir Res. 2010;11:132. doi: 10.1186/1465-9921-11-132. [PubMed: 20920222]. [PubMed Central: PMC2955663].

21. Ogasawara N, Suga K, Zaki M, Okada M, Kawakami Y, Matsunaga $\mathrm{N}$. Assessment of lung perfusion impairment in patients with pulmonary artery-occlusive and chronic obstructive pulmonary diseases with noncontrast electrocardiogram-gated fast-spin-echo perfusion MR imaging. J Magn Reson Imaging. 2004;20(4):601-11. doi: 10.1002/jmri.20150. [PubMed: 15390150].

22. Ley-Zaporozhan J, van Beek EJ. Imaging phenotypes of chronic obstructive pulmonary disease. J Magn Reson Imaging. 2010;32(6):134052. doi: 10.1002/jmri.22376. [PubMed: 21105139].

23. Ley-Zaporozhan J, Ley S, Kauczor HU. Morphological and functional imaging in COPD with CT and MRI: Present and future. Eur Radiol. 2008;18(3):510-21. doi: 10.1007/s00330-007-0772-1. [PubMed: 17899100].

24. Barbera JA, Peinado VI, Santos S. Pulmonary hypertension in chronic obstructive pulmonary disease. Eur Respir J. 2003;21(5):892-905. [PubMed: 12765440].

25. Frazier AA, Galvin JR, Franks TJ, Rosado-De-Christenson ML. From the archives of the AFIP: Pulmonary vasculature: Hypertension and infarction. Radiographics. 2000;20(2):491-524. quiz 530-1, 532. doi: 10.1148/radiographics.20.2.g00mc17491. [PubMed: 10715347].

26. Ippolito D, Querques G, Okolicsanyi S, Franzesi CT, Strazzabosco M, Sironi S. Diagnostic value of dynamic contrast-enhanced CT with perfusion imaging in the quantitative assessment of tumor response to sorafenib in patients with advanced hepatocellular carcinoma: A feasibility study. Eur J Radiol. 2017;90:34-41. doi: 10.1016/j.ejrad.2017.02.027. [PubMed: 28583645].

27. Park EA, Goo JM, Park SJ, Lee CH, Park CM. Collateral ventilation quantification using xenon-enhanced dynamic dual-energy CT: Differences between canine and swine models of bronchial occlusion. Korean J Radiol. 2015;16(3):648-56. doi: 10.3348/kjr.2015.16.3.648. [PubMed: 25995696]. [PubMed Central: PMC4435996].

28. Remy-Jardin M, Duhamel A, Deken V, Bouaziz N, Dumont P, Remy J. Systemic collateral supply in patients with chronic thromboembolic and primary pulmonary hypertension: Assessment with multidetector row helical CT angiography. Radiology. 2005;235(1):274-81. doi: 10.1148/radiol.2351040335. [PubMed: 15703314]. 